\title{
Análise das Simulações do PMIP3 Sobre o Nordeste Brasileiro Para o Período Pré-Industrial e Holoceno Médio
}

\author{
Tyhago Aragão Dias ${ }^{1}$ (D), Alexandre Araújo Costa $^{2}$, Francisco de Assis Sousa Filho ${ }^{1}$, \\ Cleiton da Silva Silveira ${ }^{1}$ \\ ${ }^{1}$ Departamento de Hidráulica e Engenharia Ambiental, Centro de Tecnologia, \\ Universidade Federal do Ceará, Fortaleza, CE, Brasil. \\ ${ }^{2}$ Mestrado Acadêmico de Ciências Físicas Aplicadas, Centro de Ciências e Tecnologia, \\ Universidade Estadual do Ceará, Fortaleza, CE, Brasil.
}

Recebido em: 19 de Abril de 2018 - Aceito em: 20 de Setembro de 2018

\begin{abstract}
Resumo
Este trabalho mostra uma analise das simulações obtidas no banco de dados do PMIP3 (Paleoclimate Modelling Intercomparison Project Phase IIII) para o período pré-industrial com os dados observados pelo CMAP (Climate Prediction Center Merged Analysis of Precipitation) e Climatic Reserch Unit (CRU) para a precipitação pluviométrica em $\mathrm{mm} / \mathrm{mês}$ e os dados de reanálise do NCEP/NCAR para a temperatura do ar a $2 \mathrm{~m}$ acima da superfície em graus Celsius, para as região do Nordeste Brasileiro (NEB) $\left(46^{\circ} \mathrm{W}-34^{\circ} \mathrm{W} ; 16^{\circ} \mathrm{S}-2^{\circ} \mathrm{S}\right)$, a fim de encontrar um conjunto de modelos com representação realista do clima do presente. Em seguida, uma comparação é feita entre os resultados das simulações desses modelos para o período pré-industrial (PI) e o Holoceno médio (HM, 6.000 anos atrás), a fim de identificar possíveis mudanças climáticas entre esses períodos sobre a América do Sul. Esses modelos do PMIP3 sugerem que houve diferenças importantes entre o clima atual e o do Holoceno Médio no que diz respeito à intensidade da monção da América do Sul (monção mais fraca no HM do que no presente).
\end{abstract}

Palavras-chave: Paleoclimatologia, clima do Holoceno médio, clima pré-industrial, PMIP, América do Sul.

\section{Analysis of PMIP3 Simulations on the Brazilian Northeast for the Pre- Industrial and Middle Holocene Period}

\begin{abstract}
The present work shows a comparison between simulation data from PMIP (Paleoclimate Modelling Intercomparison Project Phase III) for the pre-industrial climate against observations from CMAP (Climate Prediction Center Merged Analysis of Precipitation) and Climatic Reserch Unit (CRU) for precipitation and reanalysis data from NCEP/NCAR for the $2 \mathrm{~m}$ air temperature over the following regions: Northeast Brazil (NEB) $\left(46^{\circ} \mathrm{W}-34^{\circ} \mathrm{W}: 16^{\circ} \mathrm{S}-2^{\circ} \mathrm{S}\right)$, in order to find a subset of models with a realistic representation of current climate. Following, a comparison was done between results for the pre-industrial (PI) period and the mid-Holocene ( $\mathrm{MH}, \sim 6.000$ years before present), with the objective of identifying possible climatic changes between those two periods over South America. Among the twenty-two models in PMIP3 dataset, ten models were selected for analysis, since the show, for the pre-industrial period, a better representation of observed features, such as the annual cycle over the regions and spatial patterns typical of the current climatology of South America. Those PMIP3 models suggest that current climate and MH climate exhibit important differences regarding the intensity of the South America monsoon system (weaker MH monsoon compared to present).
\end{abstract}

Keywords: Paleoclimatology, mid-holocene climate, pre-industrial climate, PMIP, South America.

Autor de correspondência: Tyhago Aragão Dias, tyhago86@gmail.com. 


\section{Introdução}

Estudos sobre a evolução climática do sistema terrestre têm como objetivo entender a variabilidade natural do clima, e como a ação antrópica é capaz de interferir no sistema climático terrestre modificando o estado de equilíbrio como se conhece. Estudos paleoclimáticos oferecem a possibilidade de se compreender o comportamento do sistema climático sob uma ampla variedade de condições forçantes e mensurar a amplitude de possíveis variações naturais.

A identificação de como o clima passa de um estado de equilíbrio para outro e os mecanismos que fazem tal transição são objeto de estudo. James Croll (1821-1890) foi um dos primeiros astrofisicos a tentar relacionar os ciclos orbitais que levariam a uma era do gelo, sendo que o pesquisador Milutin Milankovitch (1879-1958), na década de 1940, mostrou que a intensidade dos efeitos da insolação variava com a latitude e a resposta do manto de gelo com as mudanças na radiação solar.

Avanços nos estudos paleoclimáticos se deram a partir de meados da década de 1970 (Imbrie e Imbrie 1979) e, hoje em dia, tem sido bastante impulsionados por investigações que subsidiam a elaboração dos relatórios do Painel Intergovernamental sobre Mudanças Climáticas (IPCC - Intergovernmental Panel on Climate Change), como no seu $5^{\circ}$ relatório (AR5, IPCC 2009), que trouxe em seu quinto capítulo do Climate Change 2013: The Physical Science Basis mostrando a relevância da incorporação dos estudos da variabilidade e dos ciclos climáticos em escalas além do último século, fenômenos de baixa frequência.

$\mathrm{Na}$ tentativa de simular o comportamento climático, a modelagem numérica entra como uma importante ferramenta de análise, podendo ser utilizada para propor hipóteses e comparar causa e efeito. O Paleoclimate Modeling Intercomparison Project (PMIP) teve seu início com o intuito de coordenar e incentivar os estudos de Modelos de Circulação Geral da Atmosfera (MCGA) aplicados ao paleoclima e mensurar sua capacidade de simular grandes variações climáticas, como as ocorridas no passado, comparando as diferentes repostas dos modelos para as mesmas condições de contorno.

Para uma melhor compreensão das simulações paleoclimaticas em comparação com o presente vamos admitir que o clima do pré-industrial (PI) é semelhante ao do presente, em sua composição continental e cobertura vegetal, modificando as concentrações de gases de efeito estufa, que altera o saldo radioativo no globo, os modelos que mostram uma capacidade de simular climas do PI similares à climatologia atual, reproduzindo os padrões de grande escala como Zona de Convergência Intertropical e Zona (ZCIT) e Convergência de Convergência do Atlântico Sul (ZCAS) em amplitude e fase, possivelmente terão uma maior probabilidade de representar períodos mais longos com maior confiabilidade.

Neste trabalho, foram analisados os resultados das simulações para o período pré-industrial do PMIP3 para as regiões do Nordeste Brasileiro (NEB) $\left(46^{\circ} \mathrm{W}-34^{\circ} \mathrm{W}\right.$ : $16^{\circ} \mathrm{S}-2^{\circ} \mathrm{S}$ ), comparando tais resultados com os dados observados do GPCP (Global Precipitation Climatology Project), Climatic Reserch Unit (CRU) e os dados observados de estações meteorologias da Fundação Cearense de Meteorologia (FUNCEME) incorporando os dados da Superintendência do Desenvolvimento do Nordeste (SUDENE), sendo estes apenas para o Estado do Ceará, para a precipitação pluviométrica e os dados de reanálise do NCEP/NCAR para a temperatura do ar a $2 \mathrm{~m}$ acima da superfície em graus Celsius. A Fig. 1 mostra as variáveis para a região de estudo, precipitação acumulada $\mathrm{em} \mathrm{mm} /$ mês para a base de dados observada, GPCP e CRU, temperatura média anual.

Tal comparação mostrará quais dos modelos são mais capazes de simular os padrões climatológicos do NEB, oferecendo possivelmente um resultado mais robusto quando se trata de avaliar mudanças climáticas passadas ou futuras. Os modelos que não mostrarem essa capacidade mínima de representar a climatologia do presente não serão considerados no presente estudo, visto que não existe garantias que os modelos mesmo tendo
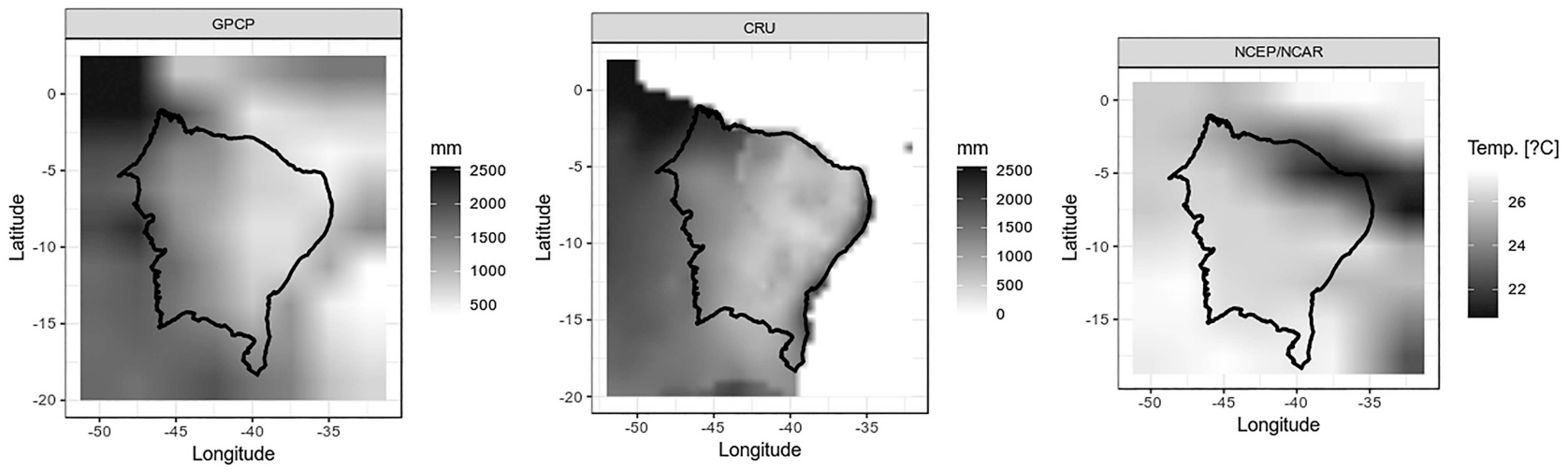

Figura 1 - Precipitação acumulada media para a base de dados dos dados observados, temperatura média anual para o NEB. 
capacidade de representar características globais o façam em aspecto regional devido a sua resolução espacial ou limitação na representação física da dinâmica interna para a região.

De posse dos modelos com melhores resultados, é realizada uma nova análise, comparando as simulações dos períodos do Holoceno médio (HM, $\sim 6,000$ anos atrás) e pré-industrial (PI). Essa comparação mostrará diferenças climáticas entre o clima desses períodos sobre o NEB, verificando indicativos de o Holoceno médio ter sido mais/menos úmido e mais/menos quente que o préindustrial.

Esses resultados de simulações podem vir a complementar e/ou a ser comparados com outros estudos de proxies (como Cruz et al. 2009), oferecendo uma visão mais completa sobre o paleoclima do NEB, cumprindo seu papel do PMIP3 em comparar causa e feito.

\section{Materiais e Métodos}

\subsection{Dados}

Os dados de modelo utilizados neste trabalho foram obtidos através do banco de dados do CMIP5/PMIP3, e que se encontra organizado por modelos, períodos de simulações e variáveis, com os arquivos em formato NETCDF. Os dados utilizados para validação dos modelos foram obtidos do GPCP (Global Precipitation Climatology Project 1979-2017), Climatic Reserch Unit (CRU 1901-2014), também em formato NETCDF todos convertidos de $\mathrm{mm} / \mathrm{s}$ para $\mathrm{mm} / \mathrm{mês}$, os dados observados da Fundação Cearense de Meteorologia (FUNCEME/ SUDENE 1911-2015) se trata de uma média de todas as estações disponíveis para o Estado do Ceará, que será utilizado na nossa analise como referência de dados instrumentais, e os dados de reanálise do NCEP/NCAR (National Center for Environmental Prediction / National Center for Atmospheric Research 1981 - 2010) temperatura do ar a $2 \mathrm{~m}$ que já se encontram em média mensal.
Podemos ver na Fig. 2 os dados precipitação pluviométrica sobre o NEB segundo, GPCP, CRU e sobre o Estado do Ceará segundo a FUNCEME/SUDENE, em boxplot para verificar os máximos e mínimos da série temporal.

\subsection{Seleção dos modelos}

Para que sejam encontrados os modelos com capacidade de representar de maneira adequada o clima corrente na região do NEB, foram feitas análises estatísticas nos dados das simulações do PI em relação aos dados observados, todos os modelos estão apresentados na Tabela 1. Calculou-se o coeficiente de correlação de Pearson entre o

Tabela 1 - Lista de modelos: Beijing Climate Centre System Model (BCC-CSM); Community Climate System Model (CCSM); Centre National de Recherches Météorologiques (CNRM); Common wealth Science and Industrial Research Organization Mark version 3 (CSIRO Mk3); Hadley Centre Global Environment Model version 2 (HadGEM2); L'Institut Pierre-Simon Laplace Coupled Model (IPSL-CM); Max Plank Institute -Earth System Model (MPI-ESM); Meteorological Research Institute Coupled General Circulation Model (MRI-CGCM).

\begin{tabular}{|c|c|c|c|}
\hline Models & $\begin{array}{l}\text { Atmos: Lat. x } \\
\text { Lon. }\end{array}$ & $\begin{array}{l}\text { Ocean: Lat. x } \\
\text { Lon. }\end{array}$ & References \\
\hline BCC-CSM-1 & T42L26 & $360 \times 384$ L40 & Wu (2012) \\
\hline CCSM4 & $0.9^{\circ} \times 1,25^{\circ} \mathrm{L} 26$ & $320 \times 384$ L60 & Gent et al. (2011) \\
\hline CNRM-CM5 & T127 x L31 & $362 \times 292$ L42 & $\begin{array}{l}\text { Voldoire et al } \\
\text { (2012) }\end{array}$ \\
\hline CSIRO-MK3-6 & $\mathrm{T} 63 \times \mathrm{L} 18$ & $192 \times 192$ L31 & Collier at al. (2011) \\
\hline HadGEM2-CC & $\begin{array}{l}1.25^{\circ} \times 1.875^{\circ} \\
\mathrm{L} 38\end{array}$ & $360 \times 216 \mathrm{~L} 40$ & Martin et al. (2011) \\
\hline HadGEM2-ES & $\begin{array}{l}1.25^{\circ} \times 1.875^{\circ} \\
\text { L38 }\end{array}$ & $360 \times 216 \mathrm{~L} 40$ & Martin et al. (2011) \\
\hline $\begin{array}{l}\text { IPSL-CM5A- } \\
\text { LR }\end{array}$ & $\begin{array}{l}1.875^{\circ} \times 3.75^{\circ} \\
\mathrm{L} 39\end{array}$ & $182 \times 149$ L31 & $\begin{array}{l}\text { Dufresne et al. } \\
\text { (2012) }\end{array}$ \\
\hline MPI-ESM-P & T63L47 & $256 \times 220 \mathrm{~L} 40$ & $\begin{array}{l}\text { Giorgetta et al. } \\
(2012)\end{array}$ \\
\hline MRI-CGCM3 & T159L48 & $364 \times 368$ L51 & $\begin{array}{l}\text { Yukimoto et al. } \\
\text { (2012) }\end{array}$ \\
\hline
\end{tabular}

Fonte: Autor.
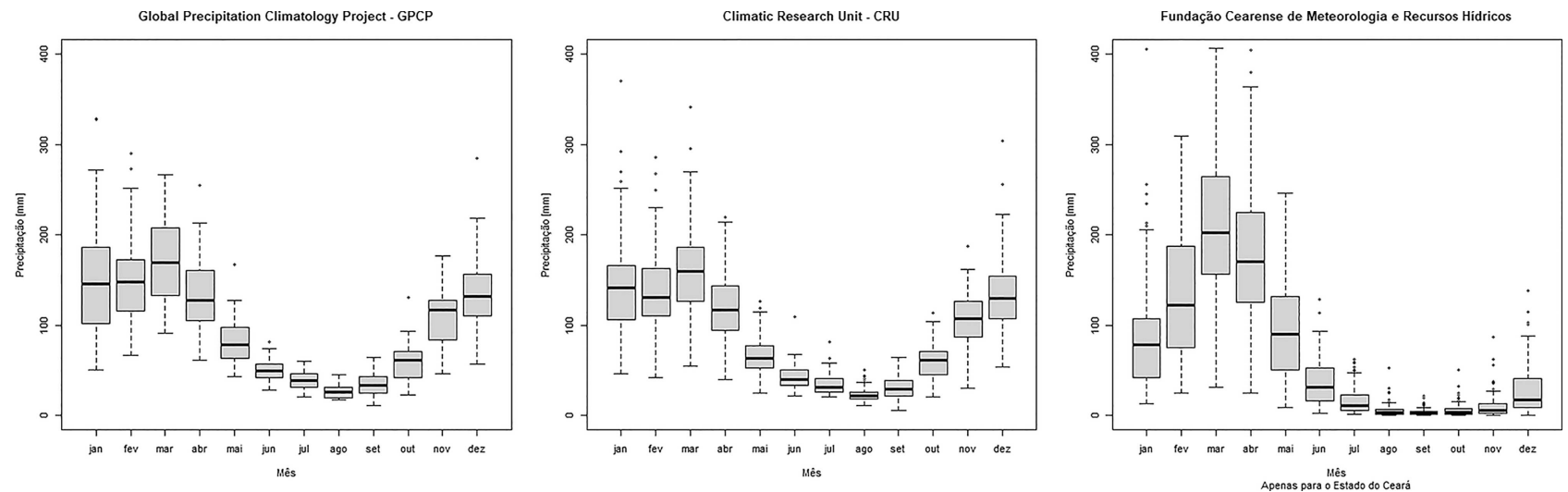

Figura 2 - Boxplot do banco de dado observado. GPCP, CRU e FUNCEM/SUDENE. 
resultado de cada simulação PI para o ciclo anual de precipitação e os dados observados. Os modelos que apresentaram a média das correlações inferior a 0,7 foram descartados, pois não tinham capacidade de representação

Também foi calculado o erro absoluto médio percentual para a precipitação climatológica mensal para cada modelo em relação ao dado observado CRU e GPCP. Aqueles modelos que mostraram uma porcentagem no acumulado anual de precipitação maior que cinquenta por cento foram desconsiderados.

\subsection{Análise dos dados}

Foi calculada a climatologia mensal (ciclo anual) para as variáveis de precipitação pluviométrica em $\mathrm{mm} /$ mês e temperatura do ar a $2 \mathrm{~m}$ acima da superfície em graus Celsius para NEB. Também foram obtidos os campos médios dessas variáveis para os períodos pré-industrial e observado e mapas de diferença entre HM e PI.

O gráfico de climatologia apresenta o resultado de cada modelo em linhas cinza, a climatologia observada, CRU e GPCP, assim como a média dos modelos, os três últimos em linha preta descontinua, para mostra a concordância entre a média dos modelos e a base de dado.

\subsection{Simulações do Pré-Industrial e Holoceno médio.}

Cada modelo tem sua própria resolução espacial e conjunto de parametrizações físicas, mas estão todos submetidos as mesmas condições de contorno propostas por Taylor et al. (2009), a Tabela 1 mostra as condições de fronteira em que todos os modelos estão submetidos, além da mesma composição dos continentes e linhas de costa, para ambas as simulações (HM e PI) estão de acordo com o presente.

A temperatura da superfície do mar (TSM), uma componente tão importante para a distribuição espacial e intensidade da precipitação, em cada modelo é calculado de maneira de independente de acordo com sua dinâmica interna de cada modelo.

A diferenças mais discernível entre as simulações HM e PI está nos parâmetros orbitais e na concentração de gases de efeito estufa, como mostrado na Tabela 2, em que (peri- $180^{\circ}$ ) é a longitude do periélio em relação ao equinócio vernal.

Tabela 2 - Resumo das condições de contorno para os experimentos.

\begin{tabular}{lcc}
\hline \multicolumn{1}{c}{ Parâmetro } & Pré-industrial & Holoceno médio \\
\hline Excentricidade & 0,016724 & 0,018682 \\
Obliquidade & $23,446^{\circ}$ & $24,105^{\circ}$ \\
Peri- $180^{\circ}$ & $102,04^{\circ}$ & $0,87^{\circ}$ \\
$\mathrm{CO}_{2}$ & Definida pelo modelo & $280 \mathrm{ppm}$ \\
$\mathrm{CH}_{4}$ & Definida pelo modelo & $650 \mathrm{ppm}$ \\
$\mathrm{N}_{2} \mathrm{O}$ & Definida pelo modelo & $270 \mathrm{ppm}$ \\
\hline
\end{tabular}

Fonte: Taylor el al. (2009), modificado.
Apenas a excentricidade altera a quantidade total de energia solar chegando ao topo da atmosfera, mas a diferença nesse parâmetro mostrada na Tabela 2 é insuficiente para produzir diferenças significativas no balanço radioativo capaz de explicar a mudança existente enter as simulações para os períodos HM e PI como será vista nos resultados. O parâmetro de precessão, produz, nessa escala de tempo (6000 anos de diferença), mudanças significativas na insolação regional no Hemisfério Sul, particularmente, vale ressaltar que, no presente, o periélio ocorre próximo ao solstício de verão austral, enquanto que, no Holoceno médio, o periélio ocorria próximo ao equinócio, com impactos importantes sobre a Monção da América do Sul, fazendo com que o sistema forçado pela obliquidade tenha uma modificação na sua sazonalidade e a excentricidade, peri- $180^{\circ}$, faça com que a monção tenha seu início em meados de setembro.

\section{Resultados e Discussões}

\subsection{Simulações do pré-industrial em comparação com observações}

Simulações do PMIP3 para o período pré-industrial que mostraram uma concordância consistente com os dados observados para a região do NEB foram analisadas considerando seus aspectos espaciais e temporais (ciclo anual) e análise estatística.

As Figs. 3 e 4 mostram, respectivamente, valores climatológicos de precipitação anual acumulada e temperatura média para as simulações PI, os campos são mostrados para os 8 modelos do PMIP3 selecionados por critério de qualidade como discutido na secção anterior. É notável que nenhum modelo mostrou uma representação perfeita, o que já era esperado.

No entanto, padrões espaciais como a presença das Zonas de Convergência Intertropical (ZCIT) e do Atlântico Sul (ZCAS) devem ser apresentados pelos modelos, o que de fato acontece, mesmo que alguns erros sistemáticos são comuns, como é o caso da representação da chuva convectiva em regiões de topografia acentuada. Por exemplo, modelos como CCSM4, HadGEM_CC, HadGEM_ES e MRI_CGCM3 tendem a superestimar a chuva nos Andes (por vezes em grandes extensões). Uma vez que os modelos estão validados no seu aspecto físico de representação da climatologia para América do Sul assim como para a região do NEB temos maior confiabilidade nos resultados.

Para a região do NEB temos os modelos HadGEM_CC, HadGEM_ES e IPSL_CM5A colocando um viés mais seco para precipitação no período PI enquanto o modelo CCSM4 concentra essa característica apenas para região sul do NEB, essa característica pode estar associada ao deslocamento da precipitação para a porção central da América do Sul, tais característica podem ser pontuadas de maneira quantitativa ao observar a Fig. 5 e as Tabela 3 


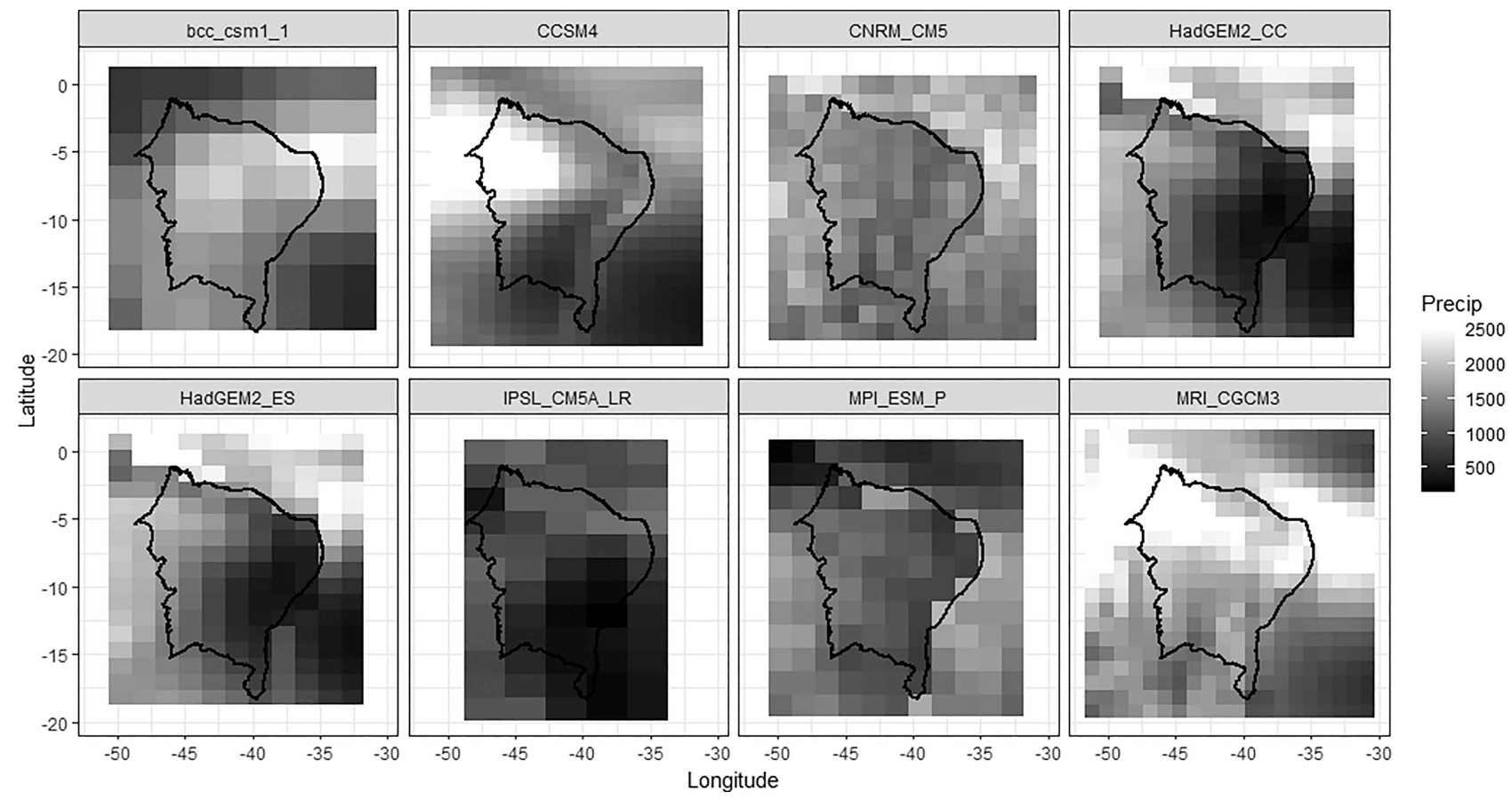

Figura 3 - Precipitação acumulado média anual.
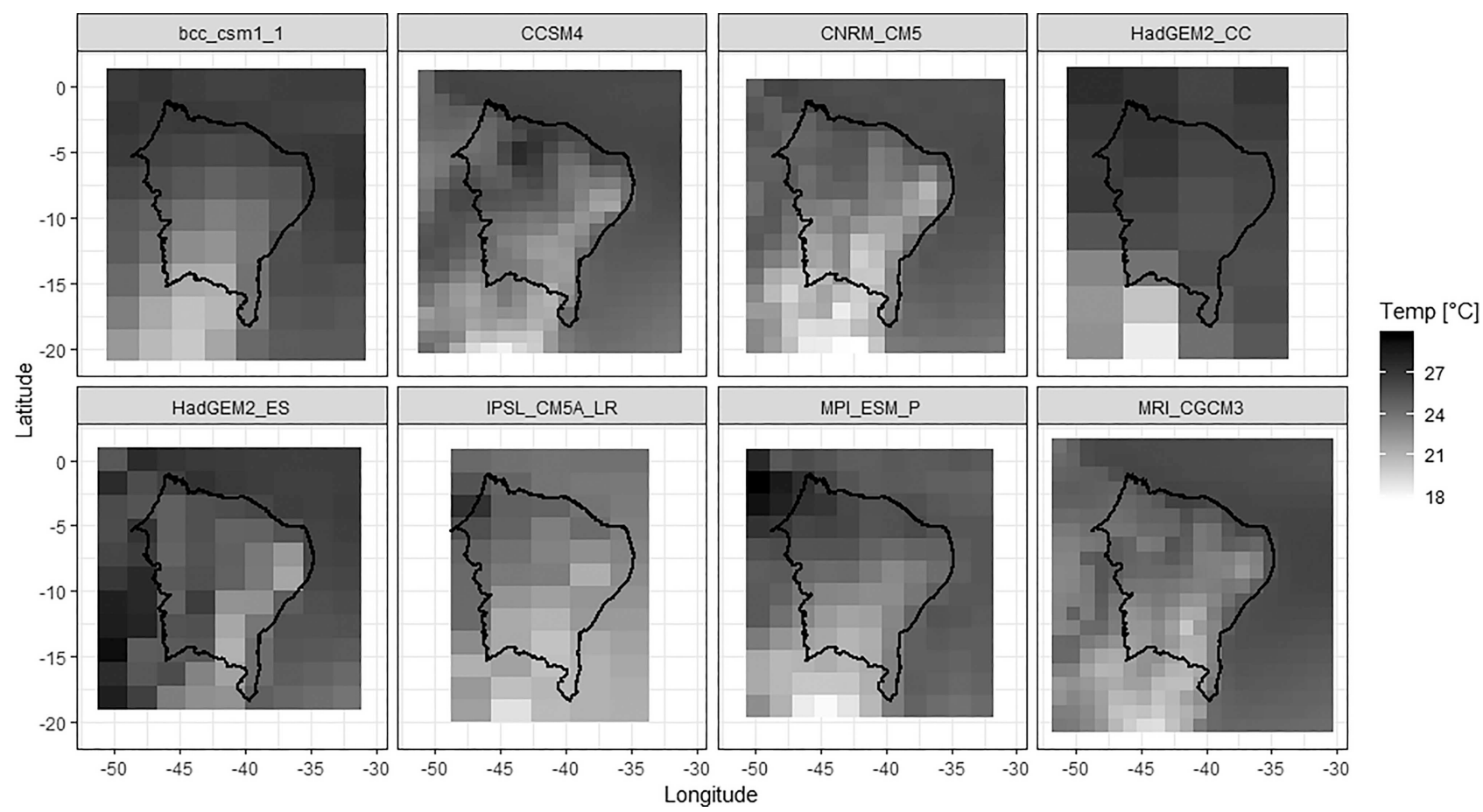

Figura 4 - Temperatura média anual.

onde mostram a correlação e o erro percentual, respectivamente, entre o ciclo anual observado (CRU e GPCP) e o simulado por cada modelo, esses dados iram corroborar na confiabilidade dos modelos em representar a região.

$\mathrm{Na}$ Fig. 5 temos esse resultado mostrando o comportamento de todos os modelos para a região do NEB, verificou-se ainda que vários modelos aprovados para análise mostram uma circulação de monção, com verão chuvoso e inverno seco (Moran e Morgan, 1986), apenas o modelo MRI_CGCM3 superestima a precipitação entre os meses de março e abril, mas no restante do ciclo anual consegue estar dentro dos valores médios esperados, essa 


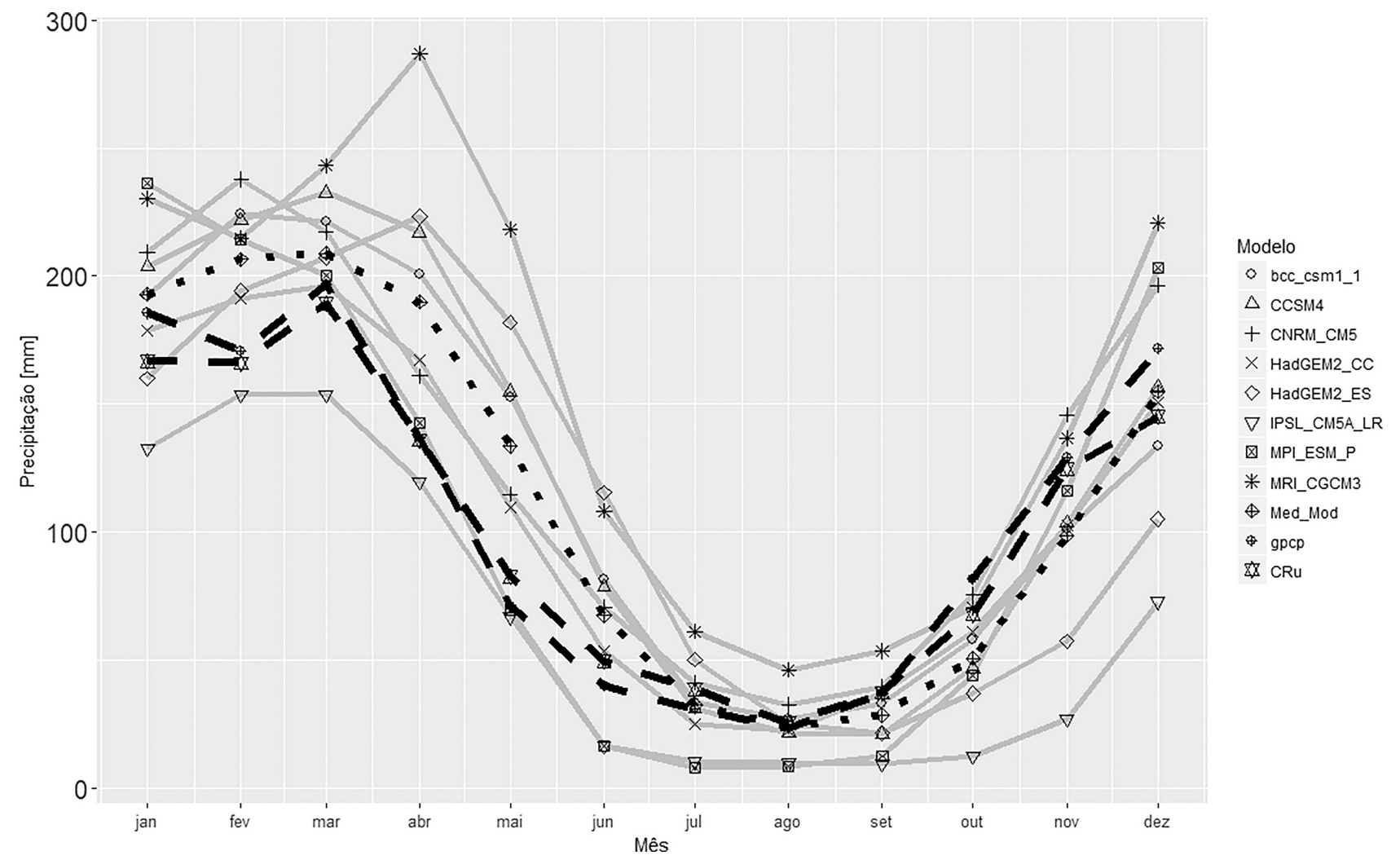

Figura 5 - Climatologia da precipitação de todos os modelos e dados observados para o NEB.

Tabela 3 - Correlação e Erro Médio Percentual (EMP) entre o dado simulado pelo modelo e o observado.

\begin{tabular}{llllll}
\hline \multirow{2}{*}{ Modelo } & \multicolumn{2}{c}{ CRU } & & \multicolumn{2}{c}{ GPCP } \\
\cline { 2 - 3 } \cline { 6 - 6 } & Correl & EMP & & Correl & EMP \\
\hline bcc_csm1_1 & 0.92 & 27.51 & & 0.86 & 29.13 \\
CCSM4 & 0.93 & 32.56 & & 0.88 & 31.52 \\
CNRM_CM5 & 0.98 & 25.47 & & 0.97 & 21.9 \\
HadGEM2_CC & 0.98 & 12.85 & & 0.95 & 14.55 \\
HadGEM2_ES & 0.74 & 38.4 & & 0.66 & 43.28 \\
IPSL_CM5A_LR & 0.9 & 36.17 & & 0.86 & 38.48 \\
MPI_ESM_P & 0.97 & 28.08 & & 0.98 & 21.52 \\
MRI_CGCM3 & 0.86 & 53.88 & & 0.82 & 50.06 \\
Med_Mod & 0.95 & 22.66 & 0.91 & 22.55 \\
GPCP & 0.99 & 8.77 & & 0 \\
CRU & 1 & 0 & 0.99 & 8.45 \\
\hline
\end{tabular}

Fonte: Autor.

habilidade dos modelos em representar o ciclo anual de precipitação para o período PI é essencial para fornecer confiabilidade em suas projeções.

Para a temperatura, como visto na Fig. 6, o modelo IPSL_CM5A mostra um viés mais frio principalmente nos meses de maio a outubro, a média dos modelos representa bem o comportamento físico da variável em relação ao ciclo hidrológico.
A precipitação acumulada para o período PI é mostrada na Fig. 4 para o conjunto de modelos. Os modelos CCSM4 e MRI_CGCM3 apresentam os maiores níveis de precipitação principalmente no estado do Maranhão, para o modelo MRI_CGCM3 esse viés mais úmido provavelmente é associado à sua superestimava nos meses mencionados, enquanto o modelo CCSM4 apresenta uma precipitação média maior durante todo o ciclo anual, mas acerta a fase durante todo o período e está dentro dos parâmetros estatísticos estipulados, resultados mostrados na Tabela 3, assim como o MRI_CGCM3, garantindo sua presença na análise.

A Fig. 7 mostra o diagrama de Taylor onde temos um resumo estatístico dos modelos para o período PI em comparação a base de dados proposta, para ambas as bases de comparação o modelo HadGEM-CC mostrou a menor aderência, apresentando a menor correlação e os maiores erro quadrático assim como o desvio padrão, mesmo assim o modelo atende aos critérios propostos para a análise. O diagrama também apresenta a boa confiabilidade que temos ao analisar a média dos modelos (Taylor. 2001).

Para a região do NEB, o maior sistema climático forçante de precipitação é a Zona de Convergência Intertropical onde exibe uma migração sazonal em torno de $14^{\circ} \mathrm{N}$ nos meses agosto/setembro e na sua posição mais ao sul em torno de $2^{\circ} \mathrm{S}$ nos meses de março/abril, a 


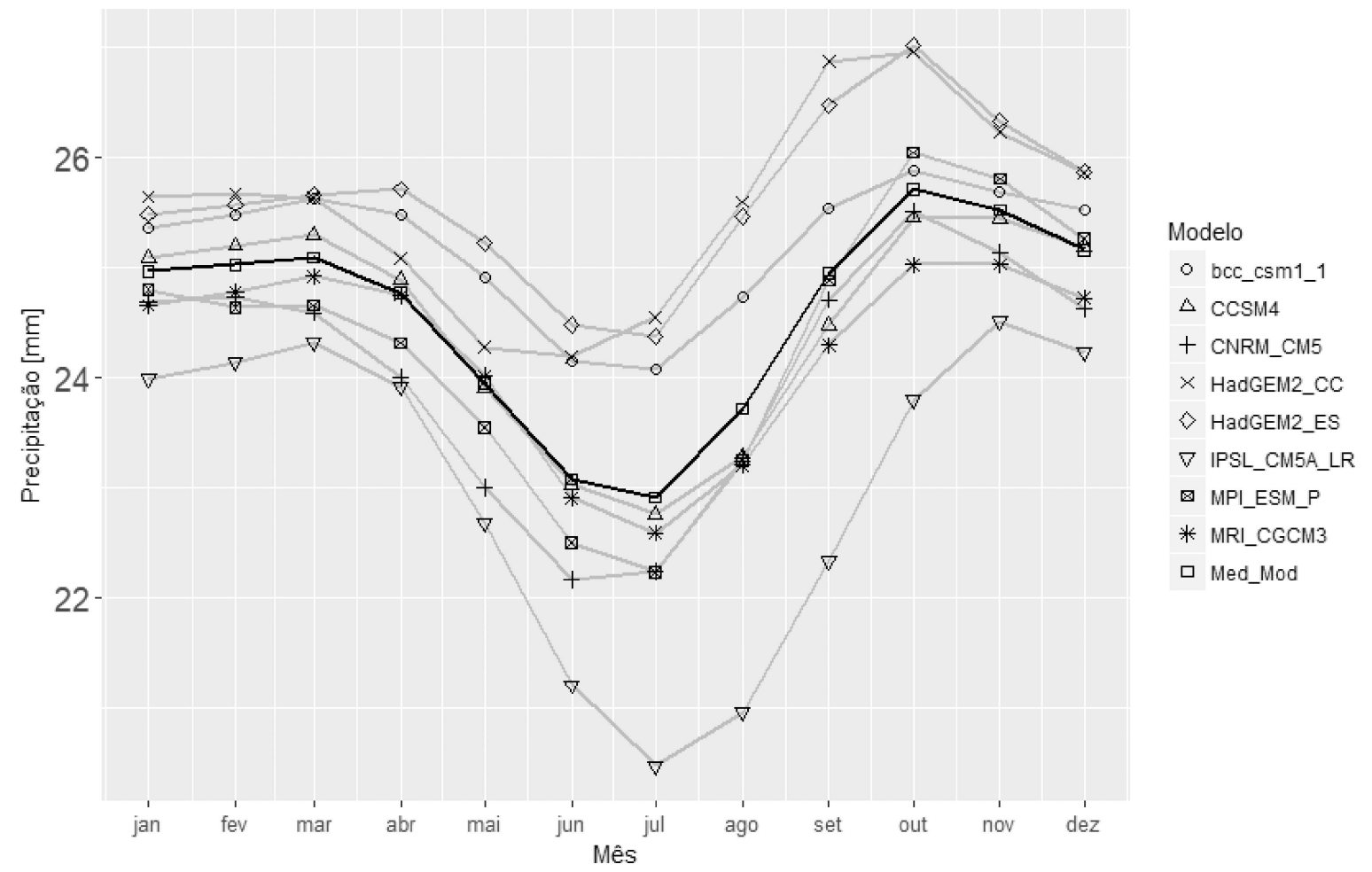

Figura 6 - Climatologia da temperatura para todos os modelos e dados observados para o NEB.
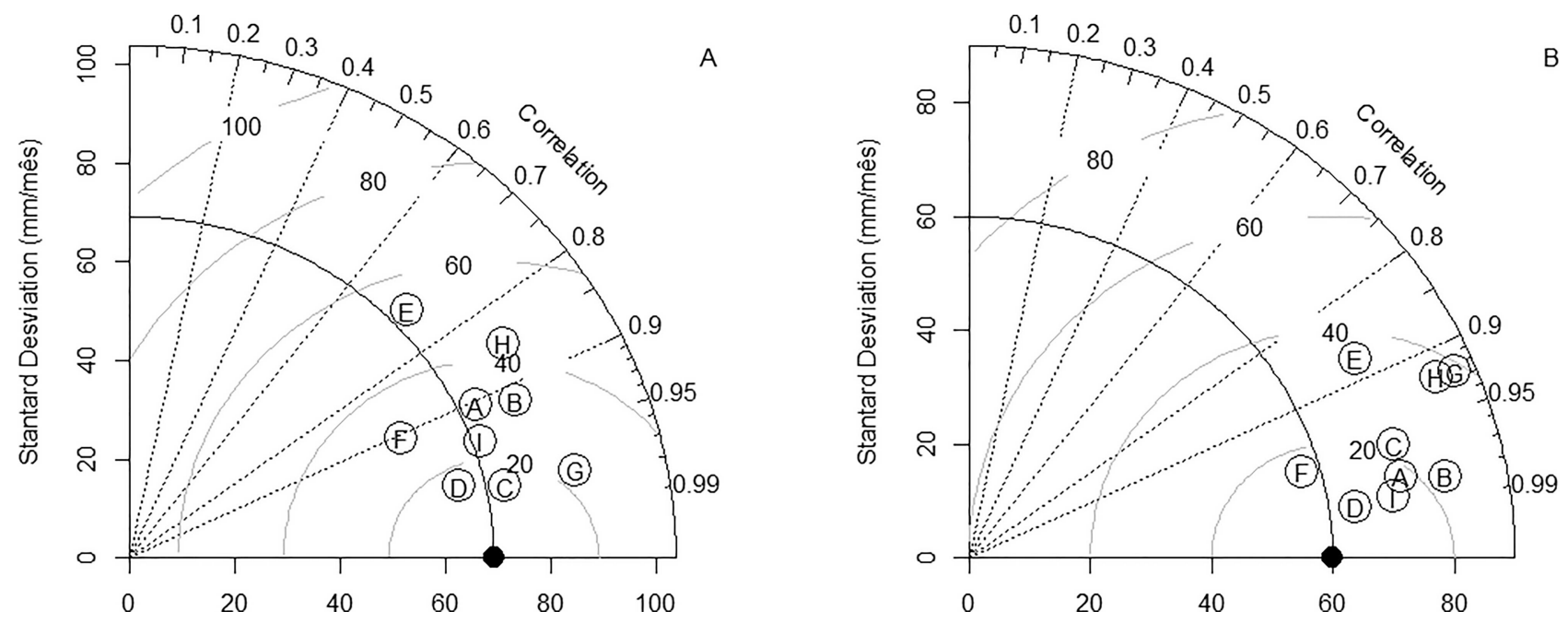

Figura 7 - Diagrama de Taylor: (A) GPCP como base de dados de referência, (B) CRU como base de dados de referência. A - bcc_csm1; B - CCSM4; C CNRM_CM5; D - HadGEM_CC; E - HadGEM_ES; F - IPSL_CM5A; G - MPI_ESM; H - MRI_CGCM3; I - Média dos modelo.

permanência da ZCIT em posições mais ao sul faz com que o NEB seja mais úmido como é sugerido por Uvo (1989).

Essa característica é importante para determinar a mudança do regime de precipitação entre os períodos simulados, entre o período de ocorrência, intensidade da precipitação e persistência da ZCIT na região do NEB.
A comparação dos observados com a média dos modelos mostra a capacidade em representar a região do NEB não existe uma grande dispersão entre o observado e o que as médias dos modelos mostram, dos meses de março a novembro as curvas estão quase que sobrepostas nos demais meses os modelos sobrestimam a precipitação, mas nada que chegue a um milímetro por dia. 


\subsection{Simulações do Holoceno médio em comparação como o Pré-Industrial}

Para realizar a comparação entre as simulações é importante entender as mudanças entre os períodos estudados, como mostrado na Tabela 2, principalmente a distribuição da radiação forçada pela mudança dos parâmetros orbitais, causando importante impacto sobre a monção da América do Sul, a composição dos continentes permanece a mesma entre os dois períodos assim como o manto de gelo que poderia interferir no feedback geloalbedo.

O que mais se destaca de diferente é a longitude do periélio em relação ao equinócio vernal. Segundo Vettoretti et al. (1998) a diferença entre a insolação do HM e do PI é devido à mudança na posição do solstício de verão para uma posição mais próxima ao periélio no HM, assim no hemisfério sul em DJF existe uma redução na insolação de $24,8 \%$ em comparação com o PI, como um aumento em JJA que não é descrito no referido artigo. Muitos autores sugerem que a região do NEB foi mais úmida no período do HM, mesmo que essa alteração na insolação para o hemisfério sul tenha enfraquecido a monção da América do Sul.

Strikis et al. (2011) apresenta resultados através de espeleotemas que mostra que a porção norte do estado de Minas Gerais foi mais úmida no HM, devido a incursões mais ao sul da ZCIT. Cane (2005) sugere que os eventos ENSO (El Niño oscilação sul) se tornaram muito raros favorecendo o NEB com precipitação, Cruz et al. (2009) também mostra através de espeleotemas que o NEB foi mais úmido que o presente.

Nossa análise converge para tais resultados em que a média dos modelos mostra que a região do NEB foi mais úmida no verão (DJF) do HM em comparação com PI, apesar que esse acréscimo se inicia em outubro, como mostrado na Fig. 8, mostra a diferença percentual da climatologia entre HM e PI.

O modelo HadGEM_ES mostra o maior gradiente entre todos os modelos, essa característica esta associada representação da climatologia do modelo onde mesmo que faça um bom trabalho em simular os aspectos físicos da região acaba colocando os máximos de precipitação nos meses vizinhos, causando tal analise.

A Fig. 6 deixa claro que os modelos têm uma tendência em amplificar as estações onde o inverno é mais seco e verão mais úmido, fazendo com que o saldo hidrológico se mantenha positivo, como pode ser visto nas proxies mostradas por (CRUZ, 2009).

Essa diferença fica mais discernível quando analisamos os campos médios em regime de precipitação sazonal, as Figs. 9 e 10 mostram respectivamente a diferença percentual entre HM e PI para o verão e inverno.

O conjunto dos modelos mostra que que cinco de oito apresentam a precipitação maior na estação de verão, corroborando as proxies, nos outros modelos a diferença não é muito grande girando em torno de zero. Para a estação de inverno os modelos CNRM_CM5 e MPI_ESM mostram um comportamento contrário a maioria dos modelos, essa característica pode ser associada a representação física e métodos de parametrização adorados pelos modelos.

A explicação mais provável para esses níveis de precipitação mais elevados no NEB para o período do Holoceno médio em relação ao Pré-Industrial se da por um mecanismo de antecipação da monção forçado pela longitude do periélio em relação ao equinócio vernal, que

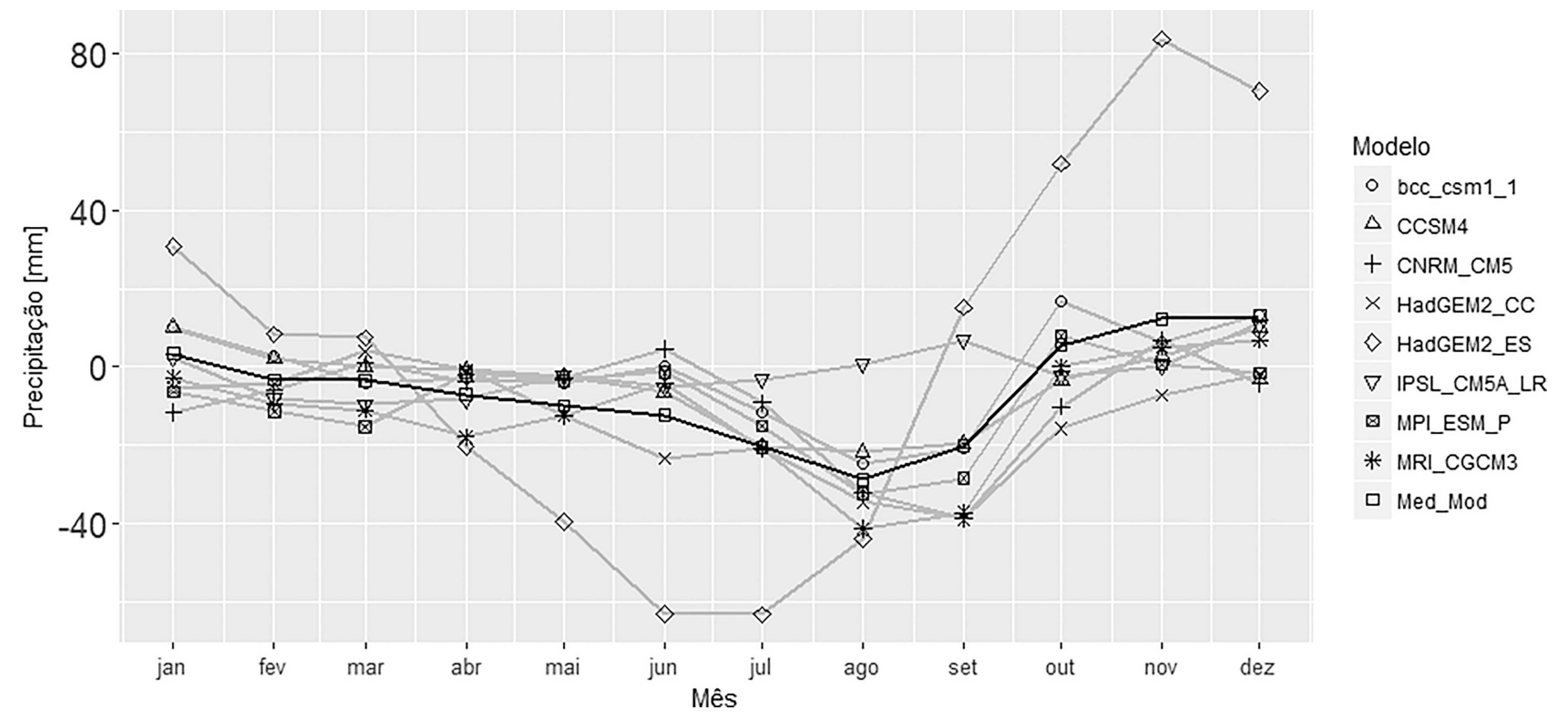

Figura 8 - Diferença percentual da climatologia de todos os modelos para o NEB. 

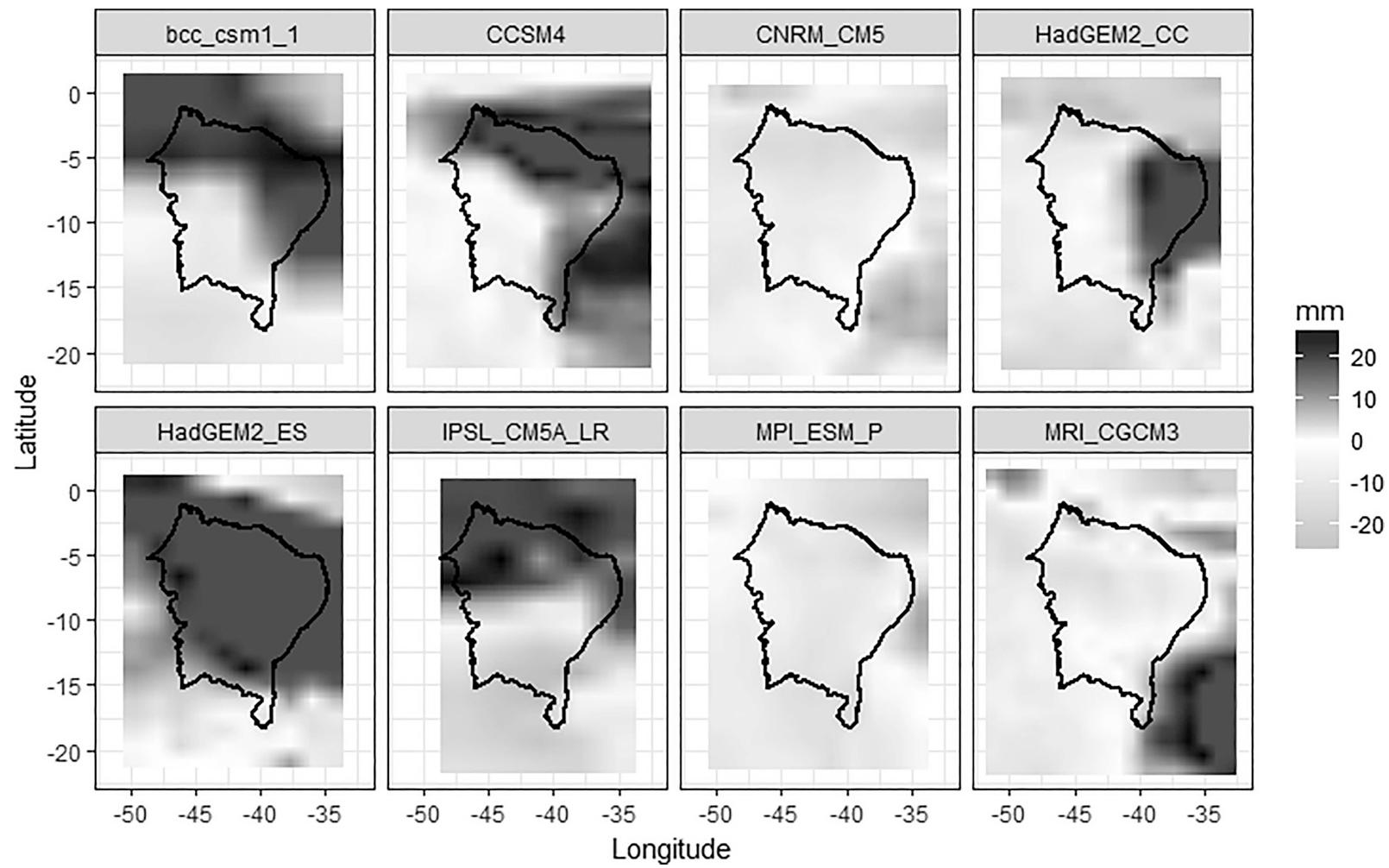

Figura 9 - Diferença percentual da precipitação para os meses Dezembro, Janeiro eFevereiro para os períodos Holoceno médio menos PI-control sobre o NEB.
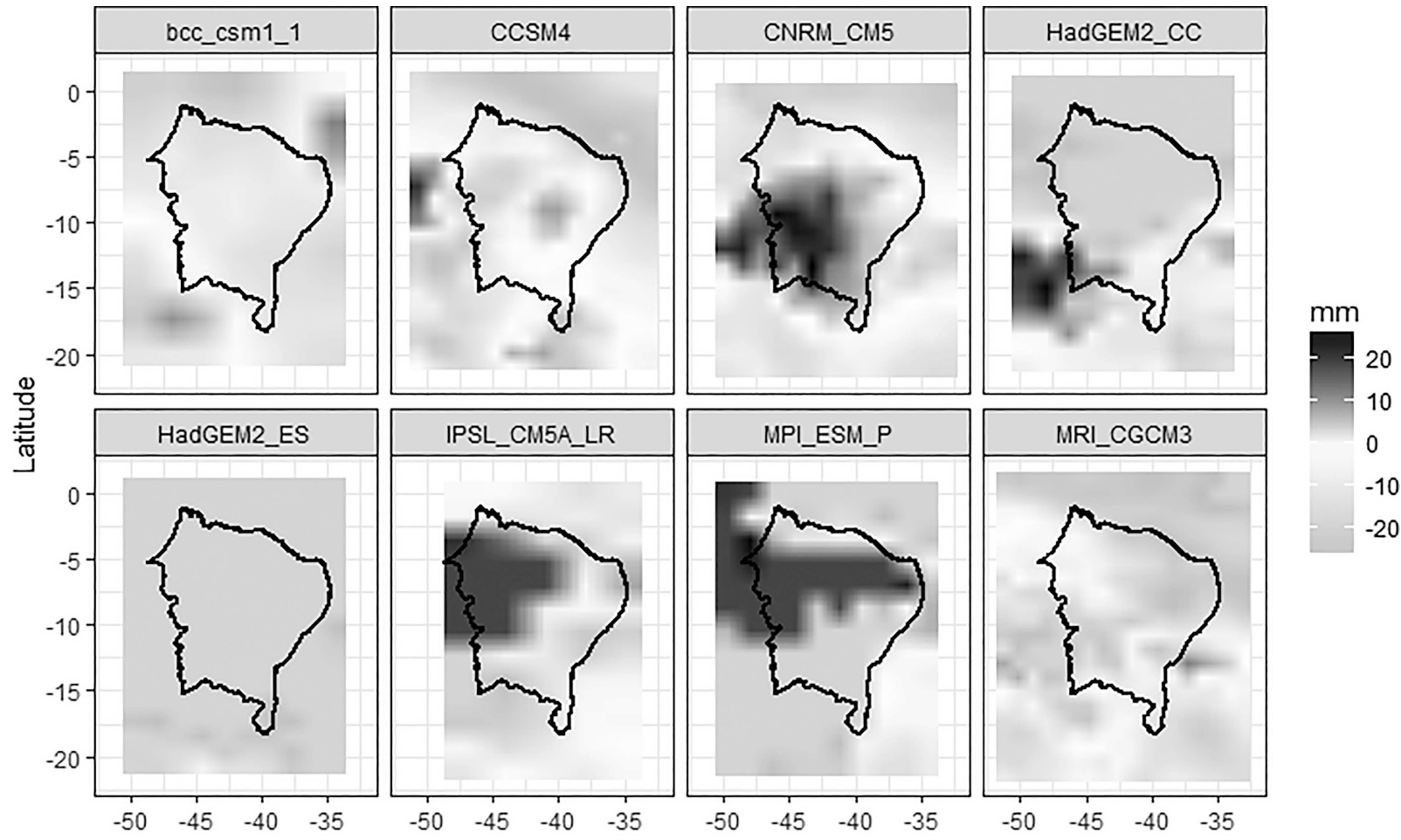

Figura 10 - Diferença percentual da precipitação para os meses Junho, Julho e Agosto para os períodos Holoceno médio menos PI-control sobre o NEB. 
causaria uma monção mais fraca porem mais persistente e com incursões mais ao Sul na porção da América do Sul.

\section{Conclusão}

As simulações do período Pré-Industrial, quando comparadas com os dados observados do GPCP, CRU e FUNCEME mostraram a capacidade dos modelos em representar a climatologia da região do NEB com um bom gral de confiabilidade, pois além de apresentar as variáveis espacialmente de maneira satisfatória ainda conseguiram reproduzir sua sazonalidade e amplitude de valores como pode ser visto ao analisado o erro absoluto médio e correlação entre modelo e observado. O método de validação dos modelos foi útil para determinar qual modelo apresentava maior aderência região visto que não se tem garantias que o mesmo modelo possa representar bem todas as porções do globo.

Esses modelos tiveram suas simulações comparadas com as do período Holoceno médio, assim observamos o quanto as variáveis de precipitação pluviométrica em milímetros por mês e temperatura média a dois metros acima da superfície em graus Celsius variou.

Os resultados obtidos foram muito conformes com os registros paleoclimático. Outros trabalhos como mostrado por Turc et al. (2002), Cane (2005), Cruz et al. (2009), Dias e Marengo (2008), Passenda et al. (2010), Strikns et al. 2011, Whitney et al. (2011), Bird et al. (2011) entre outros, mostrando que os modelos que realizam simulações paleoclimáticas tem capacidade de reconstruir o clima.

Assim o estudo de modelos paleoclimaticos tem importante papel na melhoria do entendimento do clima passado, podendo testar hipóteses e verificar mudanças ocorridas devido à forçantes de baixa frequência que podem fazendo o sistema climático terrestre entre em um novo estado de equilíbrio, principalmente devido a redistribuição da radiação.

A utilização de tais modelos será muito útil para que se possa melhorar o entendimento do clima passado, podendo testar hipóteses e verificar mudanças ocorridas devido às forçantes de mais alta frequência. Esses resultados são importantes para entender com maior clareza como ocorre as mudanças climáticas devido a ação antrópica e assim propor melhores cenários para projeções futuras.

A utilização da técnica de Downscaling dinâmico será utilizada em uma próxima etapa do trabalho, em que se espera ter um detalhamento maior da informação, levando em conta aspectos de vegetação e relevo. Essas simulações serão comparadas com as respostas dos modelos globais, podendo até mesmo inferir se tal modelo representa bem uma determinada região com o mesmo desempenho que faz em outra.

\section{Referências}

BROXTON W.B; MARK B.A; DONALD T.R; MATHIAS V. Holocene tropical South American hydroclimate revealed from a decadally resolved lake sediment $\delta 180$ record. Earth And Planetary Science Letters, v. 130, n. 3-4, p.192-202, 2011.

CANE, MARK A. The evolution of El Nino, past and future. Earth And Planetary Science Letters, v. 230, n. , p.227240, 13 Jan. 2005.

CRUZ, F. W. VUILLE, M. BURNS, S. J. WANG, X. CHENG, H. WERNER, M. EDWARDS, R. L. MARMANN, I. AULER, A. S. NGUYEN,H. Orbitally driven east-west antiphasing of South American precipitation. Nature Geoscience, v.2, n. 3, p.210-214, 2009.

MELO, M.L; MARENGO, J.A. The influence of changes in orbital parameters over South American climate using the CPTEC AGCM: simulation of climate during the mid Holocene. The Holocene, Cachoeira Paulista, v. 18, n. 4, p. 501-516, 2008.

MORAN, J.M; MORGEN, M.D. Meteorology: The atmosphere and the science of weather. Mnneapolis: Burgess Publishing, 1986.

PESSENDA, L.C.R; GOUVEIA. S.E.M; RIBEIRO, A.S; OLIVEIRA, P. E; ARAVENA, R. Late Pleistocene and Holocene vegetation changes in northeastern Brazil determined from carbon isotopes and charcoal records in soils. Palaeogeography, Palaeoclimatology, Palaeoecology, n. 297, p. 597-608, 2010.

RAO, V.B; HADA, K. Caracteristics of rainfall over Brazil. Annual variations and connections with the Southern Oscillation. Theor. Appl. Climatology, v. 42, n.2 , p. 8191, 1990.

CRUZ, F.W; CHENG, H; KARMANN, I; EDWARDS, R.L; VUILLE, M; WANG, X; DE PAULA, M.S; NOVELLO, V. F; AULER, A.S. Abrupt variations in South American monsoon rainfall during the Holocene based on a speleothem record from central-eastern Brazil. Geological Society Of America, São Paulo, v.39, n. 11, p. 1075-1078, 2011.

TAYLOR, K.E. Summarizing multiple aspects of model performance in a single diagram. Journal of Geophysical Research, v. 106, n. 7, p. 7183-7192, 2001.

TURCQ, B; ALBUQUERQUE, S.A.L; CORDEIRO; R.C; SIFEDDINE, A; SIMOES FILHO, F.F.L; SOUZA, A.G; ABRÃO, J.J; OLIVEIRA, F.B.L; SILVA, A.O; CAPITÂNEO, J. Accumulation of organic carbon in five Brazilian lakes during the Holocene. Sedimentary Geology, v. 148, n. 2, p. 319-342, 2002.

UVO, C.B; NOBRE, C.A. A Zona de Convergência Intertropical (ZCIT) e a precipitação no norte do Nordeste do Brasil. Parte I: A posição da ZCIT no Atlântico Equatorial. Climanálise, v. 4, n.7, p. 34-40, 1989.

VETTORETTI, G; PELTIER, W.R. Simulations of Mid-Holocene Climate Using an Atmospheric General Circulation Model. Journal Of Climate, v. 11,n. 10, p. 2607-2627, 1998.

WHITNEY, B.S; MAYLE, F.E; PUNYASENA, S.W; FITZPATRICK, K.A; BURN, M; GUILLEN, R; CHAVEZ, E; MANN, D; PENNINGTON, T.R; METCALFE, S.E. A 45 
kyr palaeoclimate record from the lowland interior of tropical South America. Palaeogeography, Palaeoclimatology, Palaeoecology, v. 307, n.4, p.177-192, 2011.
This is an Open Access article distributed under the terms of the Creative Commons Attribution License, which permits unrestricted use, distribution, and reproduction in any medium, provided the original work is properly cited. 